\title{
TRANSLATIONAL TRANSFORMATIONS OF TENSOR SOLUTIONS OF THE HELMHOLTZ EQUATION AND THEIR APPLICATION TO DESCRIBE INTERACTIONS IN FORCE FIELDS OF VARIOUS PHYSICAL NATURE
}

\author{
BY \\ YURI M. URMAN (Nizhny Novgorod State Pedagogical University, Nizhny Novgorod, Russia, and \\ Nizhny Novgorod Institute of Management and Business, Nizhny Novgorod, Russia) \\ AND \\ SERGEY I. KUZNETSOV (Johns Hopkins University, Baltimore, MD, USA, and Lobachevsky State \\ University of Nizhny Novgorod, Nizhny Novgorod, Russia)
}

\begin{abstract}
Using group theory and irreducible tensor formalism we derive formulas for translational transformations of the tensor solutions of the Helmholtz equation. These formulas can be used to solve different problems in theoretical and mathematical physics, where it is necessary to relate boundary conditions for two or more spatial bodies. We show that these formulas can be used to perform invariant expansions of the interaction energy of the bodies in force fields of different physical nature. These expansions have a number of advantages and are very efficient and convenient to study force interactions. Examples from celestial mechanics, space vehicle dynamics and electric current interactions are given.
\end{abstract}

1. Introduction. A number of problems in theoretical and mathematical physics require expressing a solution of the Helmholtz or Laplace equations in one coordinate system using the solutions of the same equation in another coordinate system, translated with respect to the first one. This problem arises when one needs to relate boundary conditions for two or more bodies in the problems of electrodynamics, acoustics, geodynamics, heat conduction, in quantum mechanical problems, in multipole expansions of the interaction energy for gravitating bodies, charge or current distributions, spacecraft motion, etc. For these types of problems it is necessary to know the translation operator transforming the solution from one coordinate system into another.

The transformational formulas (addition theorems) for scalar solutions of the Helmholtz equation (scalar wave functions) under translations were first obtained by

Received December 7, 2011 and, in revised form, March 14, 2012.

2000 Mathematics Subject Classification. Primary 20G45, 33C55, 35C10, 35J05, 43A90.

E-mail address: urman37@mail.ru

E-mail address: sergkuznet@hotmail.com

(C)2013 Brown University 
Friedman and Russek [1]; these results were extended by Stein [2] and Cruzan [3] on vectors and by Danos and Maximon [4] on tensor solutions. Jones [5] derived addition theorems for tensor solutions from the group theory point of view in the context of geodynamics. These derivations were based on rather tedious mathematical manipulations. Later Borghese et al. 6], Felderhof and Jones [7] presented a more compact and elegant derivation of the addition theorem for vector solutions using irreducible tensor formalism [8]. Weniger [9,10] also used irreducible tensors and spherical gradient operators; Urman [1] used irreducible tensor formalism to derive addition theorems for the general case of tensor solutions. Then a number of alternative derivations of vector addition theorems have been presented with discussion of effective calculation of the translation coefficients. Wittmann 12 derived addition theorems for scalar and vector waves using a different approach based on differential operator representations of the wave functions. Chew [13] presented an alternative derivation of the vector addition theorem using the completeness of vector wave functions and integration by parts. Mackowsky [14, Chew and Wang [15], and Kim [16, 17] rederived vector addition theorems and presented simple recurrence relations for the addition coefficients. Kim also discussed the symmetry relationships for the translation coefficients [18]. Rokhlin [19] constructed the diagonal forms of the translation operators for the scalar Helmholtz equation, which simplifies the structure of translational operators and admits stable numerical implementation. This form is important in the design of fast algorithms for computational physics methods. This theory was improved by Epton and Dembart [20]. Later, Chew [21] presented a derivation of vector and $\mathrm{He}$ and Chew of tensor 22 addition theorem with emphasis on the diagonalization of the addition theorem. Dufva et al. 23. presented a unified derivation for scalar and vector wave functions based on the concepts of radiation pattern and incoming wave pattern with a note on an efficient calculation of the translation coefficients. Yan et al. 24] presented a review of efficient methods for calculating both scalar and vector translation coefficients and studied the convergence properties of the translational addition theorem from a numerical point of view. He and Chew 25] derived the tensor addition theorem from the group theory point of view, using quantum-mechanical notation.

The applications described in the literature include electromagnetic and acoustic wave scattering (Twersky (1962) [26], Ivanov (1968) [27, Borghese (1984) 28], Borghese (2007) [29, Chew (2008) [25], Martin [31]), multipole expansions (31, 32, 33, 34, 35, 36], [37]), problems of geodynamics [5, 39] and less-known applications of finding interactions of bodies with force fields of various physical nature developed by Urman [40]. The latter is not well known despite its tremendous advantages.

In this paper, using methods from [41, we derive translational operator transforming Helmholtz equation solutions, then using irreducible tensor formalism [8], we derive addition theorems for tensor wave functions as well. The use of group theory and irreducible tensor formalism makes derivation very compact and clear, which differs from the aforementioned references [4,5] and does not use quantum-mechanical notation as in [25]. Transformations for scalar and for vector solutions of the Helmholtz equation follow as particular cases from these theorems.

In the second part of the manuscript, we give a little-known application of the addition theorem to finding force function for bodies in force fields of different physical nature, 
which allows us to solve such problems as motion of space objects, motion of rotors of non-contact gyroscopes and various devices using non-contact suspensions [40]. The force function is expressed as an invariant expansion, not related to a particular coordinate system, which is very compact and gives explicit dependence on the phase variables of the problem. It allows us to see the physical meaning of complicated interactions, to easily use symmetries of a body and force fields, and to apply asymptotic methods of nonlinear mechanics.

As examples we consider motion of a satellite in the gravitational field of a nonspherical planet, interaction of current distributions and motion of a space vehicle in terrestrial gravitational and magnetic fields.

2. Translational operator and addition theorem for the scalar solutions of the Helmholtz equation. It is known that the group of motions of space $\mathrm{E}(3)$, consisting of rotations with respect to the origin of coordinates and translations, is a symmetry group for the Helmholtz equation. It maps Helmholtz equation solutions again into its solutions. Elements of $\mathrm{E}(3)$ in $3 \mathrm{~d}$ space can be represented as elements from a set of real 4 by 4 matrices of the form 41 .

$$
g(A, \mathbf{a})=\left[\begin{array}{rrrr} 
& & & 0 \\
& A & & 0 \\
& & & 0 \\
a_{1} & a_{2} & a_{3} & 1
\end{array}\right], A \in S O_{3}, \mathbf{a}=\left(a_{1}, a_{2}, a_{3}\right) \in R^{3} .
$$

The group element $g(A, \mathbf{a})$ maps the point $\mathbf{x} \in R^{3}$ to the point $\mathbf{x} g=\mathbf{x} A+\mathbf{a} \in R^{3}$. The group product is determined by the matrix multiplication

$$
g(A, \mathbf{a}) g\left(A^{\prime}, \mathbf{a}^{\prime}\right)=g\left(A A^{\prime}, \mathbf{a} A^{\prime}+\mathbf{a}^{\prime}\right) .
$$

Geometrically, $g$ corresponds to the rotation $A$ with respect to the origin $(0,0,0) \in R^{3}$ with the following translation on the vector $\mathbf{a}$.

Let $\psi(\mathbf{r})$ be a solution of the equation $\left(\Delta+\omega^{2}\right) \psi(\mathbf{r})=0$. Its Fourier transform

$$
\psi(\mathbf{r})=\iint_{S_{2}} \exp (i \omega \mathbf{r k}) h(\mathbf{k}) d \Omega=I(h)
$$

also satisfies the Helmholtz equation. In (2.1), $\mathbf{k}$ is a unit vector $((\mathbf{k} \cdot \mathbf{k})=1)$, running over the unit sphere $S_{2}: k_{1}^{2}+k_{2}^{2}+k_{3}^{2}=1, d \Omega$ is an element of solid angle on this sphere and $h$ is an arbitrary measurable complex function on $S_{2}$ (with respect to $d \Omega$ ), such that

$$
\iint_{S_{2}} h(\mathbf{k})^{2} d \Omega(\mathbf{k})<\infty
$$

The set $L_{2}\left(S_{2}\right)$ of such functions $h$ defines a Hilbert space with scalar product

$$
\left(h_{1}, h_{2}\right)=\iint_{S_{2}} h_{1}(\mathbf{k}) h_{2}^{*}(\mathbf{k}) d \Omega(k) .
$$

Elements $g(A, \mathbf{a})$ from the group $E(3)$ act on a Helmholtz equation solution via operators $\hat{T}(g)$. Using (2.1), we can find that

$$
\hat{T}(g) \psi(\mathbf{r})=I(\hat{T}(g) h)
$$


every time $\psi=I(h)$. Operators $\hat{T}(g)$ in the space $L_{2}\left(S_{2}\right)$ are defined by the following relationships:

$$
\hat{T}(g) h(\mathbf{k})=\exp (i \omega \mathbf{a} \cdot \mathbf{k} A) h(\mathbf{k} A), g=(A, a), \quad A \in S O_{3}, a \in R^{3} .
$$

Therefore, operators $\hat{T}(g)$ acting on the functions $\psi(\mathbf{r})$ induce operators (which we also denote $\hat{T}(g))$ acting on functions $h$. It can be shown that operators (2.3) have the property of homomorphism $\hat{T}\left(g_{1} g_{2}\right)=\hat{T}\left(g_{1}\right) \hat{T}\left(g_{2}\right)$. Moreover, due to the invariance of the measure under rotation $(d \Omega(\mathbf{k} A)=d \Omega(\mathbf{k}))$, these operators are unitary in the space $L_{2}\left(S_{2}\right)$ :

$$
\left(\hat{T}(g) h_{1}, \hat{T}(g) h_{2}\right)=\left(h_{1}, h_{2}\right) .
$$

Infinitesimal operators of the Lie algebra in $L_{2}\left(S_{2}\right)$ are defined by the following relations:

$$
\begin{aligned}
& P_{1}=i \omega k_{1}, P_{2}=i \omega k_{2}, P_{3}=i \omega k_{3}, \\
& X_{1}=k_{3} \frac{\partial}{\partial k_{2}}-k_{2} \frac{\partial}{\partial k_{3}}, X_{2}=k_{1} \frac{\partial}{\partial k_{3}}-k_{3} \frac{\partial}{\partial k_{1}}, X_{3}=k_{2} \frac{\partial}{\partial k_{1}}-k_{1} \frac{\partial}{\partial k_{2}} .
\end{aligned}
$$

The relationship between these operators and group operators (2.3) is given by the expression

$$
\hat{T}(g)=\exp \left(\alpha X_{3}\right) \exp \left(\beta X_{1}\right) \exp \left(\gamma X_{3}\right) \exp (\mathbf{a} \cdot \mathbf{P}),
$$

where $\alpha, \beta, \gamma$ are the Euler angles, parametrizing the elements of the rotations group. Therefore, operators $\hat{T}(g)$ define unitary (irreducible) representation of the group $E(3)$ on the functional space $L_{2}\left(S_{2}\right)$.

Now consider a space $H$ consisting of the Helmholtz equation solutions $\psi(\mathbf{r})$ defined by the formula (2.1): $\psi(\mathbf{r})=I(h)$ for some $h \in L_{2}\left(S_{2}\right)$. The space $H$ is a Hilbert space with scalar product

$$
\left(\psi_{1}, \psi_{2}^{*}\right)=\left(h_{1}, h_{2}^{*}\right), \quad \psi_{j}=I\left(h_{j}\right) .
$$

It follows that $I$ is a unitary transformation from $L_{2}\left(S_{2}\right)$ into $H$. The existence of a unitary mapping allows us to switch in problems from the space $H$ to the space $L_{2}\left(S_{2}\right)$. In problems related to the solution of the Helmholtz equation, it is of great importance to find the formulas, giving expansions for basis functions with separating variables $\psi_{n}^{(j)}$ in one coordinate system as a sum or integral of basis functions $\psi_{m}^{(l)}$ in another coordinate system. It is often necessary to apply Euclid transformation to function $\psi_{n}^{(j)}$ and then expand it into the basis $\psi_{m}^{(l)}$. Since $H$ is a Hilbert space, we have

$$
\hat{T}(g) \psi_{n}^{(j)}=\sum_{m}\left(\hat{T}(g) \psi_{n}^{(j)}, \psi_{m}^{(l)}\right) \psi_{m}^{(l)}
$$

where the sum should be replaced with an integral if $\psi_{m}^{(l)}$ are eigenfunctions with a continuous spectrum. From formula (2.7),

$$
\left(\hat{T}(g) \psi_{n}^{(j)}, \psi_{m}^{(l)}\right)=\left(\hat{T}(g) f_{n}^{(j)}, f_{m}^{(l)}\right),
$$

where $f_{n}^{(j)}, f_{m}^{(l)}$ are the bases in the space $L_{2}\left(S_{2}\right)$ corresponding to the bases $\psi_{n}^{(j)}, \psi_{m}^{(l)}$ in the mapping $I$.

Consequently we can find expansion coefficients in the space $L_{2}\left(S_{2}\right)$ instead of finding them in the space $H$. This significantly simplifies the problem. For the case when $j=l$ and arbitrary $g \in E(3)$, expression (2.9) yields the so-called addition theorem for basis 
$\psi_{n}^{(j)}$, and coefficients $T_{n m}^{(j)}=\left(\hat{T}(g) f_{n}^{(j)}, f_{m}^{(j)}\right)$ are called matrix elements for the operator $\hat{T}(g)$ in the basis $\psi_{n}^{(j)}$.

Consider an irreducible representation $\hat{T}(g)$ of the group $E(3)$ in $L_{2}\left(S_{2}\right)$, defined by relationship (2.3). If $\hat{T}$ is restricted to the subgroup $\mathrm{SO}_{3}$, then it becomes reducible and splits on the direct sum

$$
\hat{T} \mid S O_{3} \cong \sum_{l=0}^{\infty} \oplus D_{l}
$$

where $D_{l}$ are unitary irreducible representations of the group $S_{3}$. It is known that these representations are finite-dimensional and $\operatorname{dim} D_{l}=2 l+1, l=0,1,2 \ldots$. Therefore, $L_{2}\left(S_{2}\right)$ can be expanded into a direct sum of mutually orthogonal subspaces $V_{l}$, where $\operatorname{dim} V_{l}=2 l+1$ and the action of operation $\hat{T}(A)$ on an invariant subspace $V_{l}$ is unitarily equivalent to $D_{l}$. Elements $h$ from these subspaces are eigenfunctions for the Laplace operator on the sphere $S_{2}$ and coincide with spherical functions (unit vectors of canonical basis of irreducible representation with integer weight $l$ ). Therefore, the basis for subspaces $V_{l}$ consists of the eigenfunctions

$$
f_{m}^{l}(\theta, \varphi)=Y(\theta, \varphi)=\frac{1}{\sqrt{2 \pi}} e^{i m \varphi} P_{l}^{m}(\cos \theta),
$$

where $P_{l}^{m}(\cos \theta)$ is the normalized associated Legendre function. In the following we will be using spherical functions, defined without factor $\sqrt{\frac{2 l+1}{4 \pi}}$. Spherical functions are not orthonormal with this definition; however, this simplifies many expressions which appear in the following.

Matrix elements of the translation operator $\hat{T}(E, \mathbf{a})=\exp (\mathbf{a} \cdot \mathbf{p})$ on the basis functions $L_{2}\left(S_{2}\right)$ are defined by

$$
\left.T_{l m, l^{\prime} m^{\prime}}(\mathbf{a})=\left(\hat{T}(E, \mathbf{a}) f_{m^{\prime}}^{\left(l^{\prime}\right.}\right), f_{m}^{(l)}\right)=\iint_{S_{2}} \exp (i \omega \mathbf{a} \cdot \mathbf{k}) Y_{l^{\prime} m^{\prime}}(\mathbf{k}) Y_{l^{\prime} m^{\prime}}^{*}(\mathbf{k}) d \Omega(\mathbf{k}) .
$$

To evaluate this integral we will use expansion of a plane wave

$$
e^{i \mathbf{k r}}=\sum_{l, m} i^{l}(2 l+1) J_{l}(\mathbf{k r}) Y_{l m}(\mathbf{k}) Y_{l m}^{*}(\mathbf{r})
$$

and value of the integral

$$
\int Y_{l m}^{*}(\mathbf{k}) Y_{l_{1} m_{1}}(\mathbf{k}) Y_{l_{2} m_{2}}(\mathbf{k}) d \Omega=C_{l_{1} 0 l_{2} 0}^{l 0} C_{l_{1} m_{1} l_{2} m_{2}}^{l m},
$$

where $J_{l}(\mathbf{k r})$ are the Bessel spherical functions, and $C_{l_{1} m_{1} l_{2} m_{2}}^{l m}$ are Clebsch-Gordan coefficients for $\mathrm{SO}_{3}[$ []. Then we will have

$$
\begin{gathered}
\left(\hat{T}(E, \mathbf{a}) Y_{l^{\prime} m^{\prime}}, Y_{l m}\right)=\sum_{s, q} i^{s}(2 s+1) J_{s}(\omega \mathbf{a}) Y_{s q}^{*}(\mathbf{a}) \int Y_{s q}(\mathbf{k}) Y_{l^{\prime} m^{\prime}}(\mathbf{k}) Y_{l m}(\mathbf{k}) d \Omega \\
=\sum_{s, q} i^{s}(2 s+1) J_{s}(\omega a) C_{s 0 l^{\prime} 0}^{l 0} C_{s q l^{\prime} m^{\prime}}^{l m} Y_{s q}^{*}\left(\Omega_{a}\right) .
\end{gathered}
$$

Therefore, matrix elements of the translation operator have the form

$$
T_{l m, l^{\prime} m^{\prime}}(\mathbf{a})=\sum_{s, q} i^{s}(2 s+1) J_{s}(\omega a) C_{s 0 l^{\prime} 0}^{l 0} C_{s q l^{\prime} m^{\prime}}^{l m} Y_{s q}^{*}\left(\Omega_{a}\right) .
$$


Matrix elements (2.11) can be used to obtain an addition theorem for solutions of the Helmholtz equation in a spherical coordinate system. Using formulas (2.8) and (2.9), we will have

$$
\psi_{M}^{L}(\mathbf{R})=\sum_{l, m} T_{l m, L M}(\mathbf{a}) \psi_{m}^{l}(\mathbf{r}), \quad \mathbf{R}=\mathbf{r}+\mathbf{a} .
$$

The addition theorem can be explicitly expressed as an expansion of a solution in bipolar harmonics (irreducible tensor product of spherical functions with different arguments).

$$
\begin{gathered}
Z_{j}(\omega R) Y_{j M}\left(\Omega_{R}\right)=\sum_{l, Q} i^{l+Q-j} \frac{(2 l+1)(2 Q+1)}{2 j+1} C_{l 0 Q 0}^{j 0} J_{l}(\omega a) Z_{Q}(\omega r)\left\{Y_{l}\left(\Omega_{a}\right) \otimes Y_{Q}\left(\Omega_{r}\right)\right\}_{j M}, \\
a<r .
\end{gathered}
$$

Here $Z_{L}(\omega r)$ is any spherical Bessel function. In the case when $a>r$, one has to switch $a$ and $r$ in (2.13). The expression in the curly brackets is an irreducible tensor which can be expanded as 8 ]

$$
\left\{Y_{l} \otimes Y_{Q}\right\}_{j M}=\sum_{m, n} C_{l m Q n}^{j M} Y_{l m} Y_{Q n} .
$$

Choosing the spherical Bessel function $j_{L}(\omega r)$ for $Z_{L}(\omega r)$ in (2.13) and limiting $\omega \rightarrow 0$ taking into account that $j_{L}(x) \Rightarrow \frac{2^{L} L !}{(2 L+1)} x^{L}$, we will have translation transformations for the solutions of the Laplace equation without singularities at zero

$$
\Im_{j}(\mathbf{r}+\mathbf{a}=\mathbf{R})=\sum_{\substack{l, Q=0 \\ l+Q=j}}^{\infty} \sqrt{\frac{(2 j) !}{(2 l) !(2 Q) !}}\left\{\Im_{l}(\mathbf{a}) \otimes \Im_{Q}(\mathbf{r})\right\}_{j} .
$$

Similarly, choosing a Neumann function $n_{L}(\omega r)$ and limiting $\omega \rightarrow 0$, taking into account that $n_{L}(\omega r) \Rightarrow-(2 L-1) ! ! x^{-(L+1)}$ gives the solutions of the Laplace equation with singularities in zero

$$
\Re_{j}(\mathbf{R})=\sum_{\substack{l, Q=0 \\ Q-l=j}}^{\infty} \sqrt{\frac{(2 Q+1) !}{(2 j+1) !(2 l) !}}\left\{\Im_{l}(\mathbf{r}) \otimes \Re_{Q}(\mathbf{a})\right\}_{j}
$$

In formulas (2.14) and (2.15),

$$
\begin{aligned}
& \Im_{n}(\mathbf{r}) \rightarrow \Im_{n m}(\mathbf{r})=r^{n} Y_{n m}\left(\Omega_{r}\right), \\
& \Re_{n}(\mathbf{r}) \rightarrow \Re_{n m}(\mathbf{r})=r^{-(n+1)} Y_{n m}\left(\Omega_{r}\right)
\end{aligned}
$$

are the regular and irregular (corresponding to their behavior at the point $r=0$ ) solid spherical harmonics.

3. Addition theorem for the tensorial spherical waves. Tensor spherical functions are the solutions of the Helmholtz equation of the form

$$
Z_{L}(\mathbf{k r}) Y_{j M}^{L S}(\theta, \varphi)
$$


where $Z_{L}(\mathbf{k r})$ is any spherical Bessel function, and

$$
Y_{j M}^{L S}(\theta, \varphi)=\left\{Y_{L} \otimes \chi_{S}\right\}_{j M}=\sum_{m, \mu} Y_{L m} \chi_{S \mu} C_{L m S \mu}^{j M}
$$

is a spherical tensor [8], which is an irreducible tensor product of rank $l$ of spherical and spin functions.

Particular solutions for the scalar (when $S=0$ ) and particular solutions for the vector (when $S=1$ ) Helmholtz equation follow from the equation (3.2). Choosing the spherical Bessel function $j_{L}(\mathbf{k r})$ or Neumann function $n_{L}(\mathbf{k r})$ as $Z_{L}(\mathbf{k r})$ and making limit transition $k \rightarrow 0$ yields particular tensor solutions of the Laplace equation $r^{L} Y_{j M}^{L S}(\theta, \varphi)$ with a singularity at infinity and $r^{-(L+1)} Y_{j M}^{L S}(\theta, \varphi)$ with singularity at zero. We will denote them correspondingly as

$$
\Im_{j M}^{L S}(\mathbf{r})=r^{L} Y_{j M}^{L S}(\theta, \varphi), \Re_{j M}^{L S}(\mathbf{r})=r^{-(L+1)} Y_{j M}^{L S}(\theta, \varphi) .
$$

To derive transformational formulas for translations of tensor Helmholtz equation solutions $Z_{L}(\mathbf{k r}) Y_{j M}^{L S}$, we will make use of a change in the coupling scheme in irreducible tensor products [8] and addition theorem for scalar waves. Sequentially we will have

$$
\begin{aligned}
& Z_{L}(\omega \mathbf{R}) Y_{j M}^{L S}\left(\Omega_{R}\right)=Z_{L}(\omega \mathbf{R})\left\{Y_{L}\left(\Omega_{R}\right) \otimes \chi_{S}\right\}_{j M}=\left\{Z_{L}(\omega \mathbf{R}) Y_{L}\left(\Omega_{R}\right) \otimes \chi_{S}\right\}_{j M} \\
& =\sum i^{l+Q-L \frac{(2 l+1)(2 Q+1)}{2 j+1}} C_{l 0 Q 0}^{L 0} J_{L}(\omega \mathbf{a}) Z_{Q}(\omega \mathbf{r})\left\{\left\{Y_{l}\left(\Omega_{a}\right) \otimes Y_{Q}\left(\Omega_{r}\right)\right\}_{L} \otimes \chi_{S}\right\}_{j M} .
\end{aligned}
$$

Changing the coupling scheme for an irreducible tensor product in (3.4), we will have

$$
\begin{aligned}
& \left\{\left\{Y_{l}\left(\Omega_{a}\right) \otimes Y_{Q}\left(\Omega_{r}\right)\right\}_{L} \otimes \chi_{S}\right\}_{j} \\
& =(-1)^{l+Q+j+S} \sum_{h} \sqrt{(2 h+1)(2 L+1)}\left\{\begin{array}{ccc}
l & Q & L \\
S & j & h
\end{array}\right\} \times\left\{Y_{l}\left(\Omega_{a}\right) \otimes\left\{Y_{Q}\left(\Omega_{r}\right) \otimes \chi_{S}\right\}_{h}\right\}_{j} \\
& =(-1)^{j+S-L} \sum_{h} \sqrt{(2 h+1)(2 L+1)}\left\{\begin{array}{ccc}
Q & l & L \\
S & j & h
\end{array}\right\}\left\{Y_{l}\left(\Omega_{a}\right) \otimes Y_{h}^{Q S}\left(\Omega_{r}\right)\right\}_{j} .
\end{aligned}
$$

Substituting (3.5) into (3.4) gives the resulting transformation rule for spherical tensor waves under translation:

$$
\begin{aligned}
& Z_{L}(\omega \mathbf{R}) Y_{j M}^{L S}\left(\Omega_{R}\right)=\sum_{Q, h, l} i^{l+Q-L}(-1)^{S+Q-j}(2 l+1) \sqrt{(2 h+1)(2 Q+1)} C_{l 0 L 0}^{Q 0} \\
& \times\left\{\begin{array}{ccc}
L & l & Q \\
h & S & j
\end{array}\right\} J_{l}(\omega \mathbf{a}) Z_{Q}(\omega \mathbf{r})\left\{Y_{l}\left(\Omega_{a}\right) \otimes Y_{h}^{Q S}\left(\Omega_{r}\right)\right\}_{j M} .
\end{aligned}
$$

Similarly to the translational rule for scalar solutions of the Laplace equation, we get the rules for the tensor solution of the Laplace equation.

$$
\Im_{j M}^{L S}(\mathbf{R})=\sum_{\substack{l, Q, h \\
l+Q=h}}^{\infty}(-1)^{S+l+h} \sqrt{\frac{(2 L+1) !(2 h+1) !}{(2 l) !(2 Q) !}}\left\{\begin{array}{lll}
l & Q & L \\
j & S & h
\end{array}\right\}\left\{\Im_{h}^{l S}(\mathbf{r}) \otimes \Im_{Q}(\mathbf{a})\right\}_{j M}
$$




$$
\Re_{j M}^{L S}(\mathbf{R})=\sum_{\substack{l, Q, h \\
l+Q=h}}^{\infty}(-1)^{S+l+h} \sqrt{\frac{(2 L+1) !(2 h+1) !}{(2 l) !(2 Q) !}}\left\{\begin{array}{lll}
l & Q & L \\
j & S & h
\end{array}\right\}\left\{\Im_{h}^{l S}(\mathbf{r}) \otimes \Re_{Q}(\mathbf{a})\right\}_{j M} .
$$

In these expressions $\left\{\begin{array}{ccc}l & Q & L \\ S & j & h\end{array}\right\}$ are $6 j$ Wigner symbols 8 .

4. Invariant expansion of the force function for spatial mass or charge distributions. The addition theorems derived above may be used in various problems of mathematical physics, where it is required to relate boundary conditions for two or more bodies (diffraction on many bodies, heat conduction problems, diffusion problems, etc.). In [42, an important problem of celestial mechanics on the invariance of the expansion of interaction energy for $N$ spatial gravitating bodies was considered. The same approach is applicable for describing the interaction of electric charge distributions. In this section we will present an invariant expansion of the force function for mutual gravitational attraction between two bodies of arbitrary shape; it demonstrates the application of the scalar addition theorem. In the next section we will consider an example of satellite motion in the gravitational field of a non-spherical planet.

From potential theory it is known that the force function $V_{G}$ of mutual gravitational attraction between two bodies $v_{1}$ and $v_{2}$ is

$$
V_{G}=f \int_{v_{2}} d v_{2} \int_{v_{1}} d v_{1} / \Delta=\int_{v_{2}} \bar{V}(2) d v_{2},
$$

where $\bar{V}=f \int_{v_{1}} d v_{1} / \Delta$ is the force function of body $v_{1}$ in the gravitational field of a particle of unit mass located at the point 2 and belonging to body $v_{2} ; \Delta$ is the distance between particles of the bodies $v_{1}$ and $v_{2}$, and $f$ is the gravitational constant. Denote by $r_{i}$ the radius vectors of the particles of the body $v_{i}$ with respect to the center of the body $v_{i}$, and by $\mathbf{R}$ the radius-vector of the center of body $v_{2}$ relative to that of body $v_{1}$ (Fig. 1). Then

$$
\Delta=\left|\mathbf{r}_{1}-\mathbf{a}\right|,
$$

where $\mathbf{a}=\mathbf{r}_{2}+\mathbf{R} ; r_{1}, r_{2}<R$.

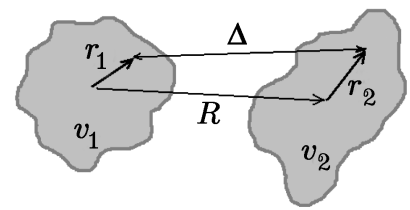

FIG. 1. Two gravitating bodies of arbitrary shape

To find the expression for $\bar{V}(2)$ we use the well-known series

$$
\frac{1}{\Delta}=\frac{1}{\left|\mathbf{r}_{1}-\mathbf{a}\right|}=\sum_{l=1}^{\infty}\left(\Im_{l}\left(\mathbf{r}_{1}\right) \cdot \Re_{l}(\mathbf{a})\right)
$$


where $\Im_{l}\left(\mathbf{r}_{1}\right)$ and $\Re_{l}(\mathbf{a})$ are regular and irregular (by their behavior in the point $r=0$ ) solid spherical harmonics, defined in (2.16); they correspond to the case $S=0$ in (3.3). The expression in the parentheses in (4.3) is a scalar product of two irreducible tensors.

Substituting (4.3) in the integral for $\bar{V}$ gives

$$
\bar{V}=f \sum_{l=1}^{\infty}\left(I_{l}(1) \cdot \Re_{l}(\mathbf{a})\right),
$$

where

$$
I_{l}(1)=\int_{v_{1}} \Im_{l}\left(\mathbf{r}_{1}\right) d v_{1}
$$

is an irreducible tensor of rank $l$; its physical meaning will be clarified later. Substituting (4.4) in (4.1) yields

$$
V_{G}=f \sum_{l=0}^{\infty}\left(I_{l}(1) \cdot \int_{v_{2}} \Re_{l}(\mathbf{a}) d v_{2}\right) .
$$

To evaluate the integral in (4.6) we use addition theorem (3.8) with $S=0$ or its particular form for scalar fields (2.15),

$$
\Re_{l}(\mathbf{a})=\Re_{l}\left(\mathbf{r}_{2}+\mathbf{R}\right)=\sum_{n=0}^{\infty} \sqrt{\frac{(2 l+2 n+1) !}{(2 l+1) !(2 n) !}}\left\{\Im_{n}\left(\mathbf{r}_{2}\right) \otimes \Re_{n+l}(\mathbf{R})\right\}_{l},
$$

where the expression in the parentheses is a tensor product of irreducible tensors of rank $n$ and $l$. Substituting (4.7) in (4.6) after integration, we have

$$
V_{G}=f \sum_{l, n} \sqrt{\frac{(2 l+2 n+1) !}{(2 l+1) !(2 n) !}}\left(I_{l}(1) \cdot\left\{I_{n}(2) \otimes \Re_{n+l}(\mathbf{R})\right\}_{l}\right) .
$$

Applying the change of coupling scheme for irreducible tensors [8], we find more symmetric expression for the force function

$$
V_{G}=f \sum_{l, n}(-1)^{n} \sqrt{\frac{(2 l+2 n) !}{(2 l) !(2 n) !}}\left(\left\{I_{l}(1) \otimes I_{n}(2)\right\}_{l+n} \cdot \Re_{n+l}(\mathbf{R})\right) .
$$

The phase factor in (4.9) depends on the direction of the vector $\mathbf{R}$. If $\mathbf{R}$ points from body $v_{2}$ toward body $v_{1}$, then in (4.9) $(-1)^{n}$ should be replaced with $(-1)^{l}$, because $\Re_{n+l}(-\mathbf{R})=(-1)^{n+l} \Re_{n+l}(\mathbf{R})$.

We should emphasize that in deriving (4.8) and (4.9) we didn't introduce any coordinate system, and the form of expansion (scalar product of two invariant objects) shows that every term of the expansion is an invariant object, independent of any coordinate system. Every term is (4.8) can be considered as an interaction described by a scalar product of a tensor related to the body $v_{1}$ with gravitational field of body $v_{2}$, defined by tensors $\left\{I_{n}(2) \otimes \Re_{n+l}(\mathbf{R})\right\}_{l}$. There is a more common in physics interpretation of formula (4.9) in terms of multipoles interaction: it describes the interaction of multipoles of different orders associated with the bodies $v_{1}$ and $v_{2}$.

Series (4.8) and (4.9) converge absolutely and uniformly if $R>\max \left(\mathbf{r}_{1}+\mathbf{r}_{2}\right)$, where $\max \left(\mathbf{r}_{1}+\mathbf{r}_{2}\right)$ is the maximum of distance from the centers of bodies $v_{1}$ and $v_{2}$ to their surfaces. 
We will write here the first few terms of the expansion (4.9) assuming that the centers of both bodies coincide with their centers of inertia with precision up to the fourth power of the ratio of the biggest linear dimension to the distance between their center:

$$
\begin{aligned}
& V_{G}=f \frac{m_{1} m_{2}}{R}+f \frac{m_{1}}{R^{3}}\left(I_{2}(2) \cdot Y_{2}(\hat{\mathbf{R}})\right)+f \frac{m_{2}}{R^{3}}\left(I_{2}(1) \cdot Y_{2}(\hat{\mathbf{R}})\right) \\
& +f \frac{m_{1}}{R^{4}}\left(I_{3}(2) \cdot Y_{3}(\hat{\mathbf{R}})\right)+f \frac{m_{2}}{R^{4}}\left(I_{3}(1) \cdot Y_{3}(\hat{\mathbf{R}})\right) \\
& +f \frac{m_{1}}{R^{5}}\left(I_{4}(2) \cdot Y_{4}(\hat{\mathbf{R}})\right)+f \frac{m_{2}}{R^{5}}\left(I_{4}(1) \cdot Y_{4}(\hat{\mathbf{R}})\right)+f \frac{\sqrt{70}}{R^{5}}\left(\left\{I_{2}(1) \otimes I_{2}(2)\right\}_{4} \cdot Y_{4}(\hat{\mathbf{R}})\right) \\
& +\ldots
\end{aligned}
$$

The force function expansion was performed with the same accuracy in the work by A. A. Orlov [43, where the expansion was found using another method. It has a different, non-invariant, and a rather tedious form. Due to its invariance, expression (4.10) undoubtedly is more preferable.

5. Example: Satellite motion in the gravitational field of a non-spherical planet. Consider the motion of a satellite in the field of a planet. Assume that the satellite (body $v_{2}$ ) has a spherical shape with homogeneous or spherical density. The planet (body $v_{1}$ ) is assumed to be non-spherical; for example, the earth is non-spherical, its gravitational shape, that of a geoid, has a number of terms in its expansion in spherical functions [44, and its animated image can be found in [45].

Since $v_{2}$ is spherical, then all $I_{n}(2)$ for $n>0$ are equal to zero and the force function reduces to

$$
V_{G}=f m_{2} \sum_{l}\left(I_{l}(1) \cdot \Re(\mathbf{R})\right)=f m_{2} \sum_{l=0} v_{l} .
$$

Formula (5.1) yields the force function describing the attraction of a sphere by a nonspherical body. In the problem of motion of a satellite in the gravitational field of a non-spherical planet, it is necessary to transform the force function to the osculating variables. The representation of the force function in terms of a scalar product of two irreducible tensors allows easy transforming to the Kepler elements.

To describe the satellite's motion we will introduce the following coordinate systems: $C X Y Z$ is an inertial coordinate system placed at the center of the planet $C$ (the $Z$ axis coincides with the rotation axis of the planet, axes $X$ and $Y$ are placed in the equatorial plane, and the $X$ axis points to the vernal equinox); $C \xi \eta \zeta$ is a coordinate system associated with the planet and spinning about axis $Z$ with angular velocity $\omega_{0}$ ( $\lambda$ is Greenwich sidereal time); $O X_{1} X_{2} X_{3}$ is a perigee coordinate system (the $X_{3}$ axis is directed along the normal to the orbital plane, the $X_{1}$ axis is directed along the radius vector of the orbit's perigee, and the $X_{2}$ axis is directed along the tangent in the perigee orbit toward the moving direction of the satellite's centre of mass).

The orientation of the coordinate system $O X_{1} X_{2} X_{3}$ with respect to the coordinate system $C X Y Z$ is defined by the following angles: $\Omega$ (longitude of ascending node of the orbit from the vernal equinox), $I$ (inclination of orbit to the equator), $\omega_{p}$ (perigee argument). The orientation of the satellite's radius vector in the perigee coordinate system is defined by the spherical angles $\theta=\pi / 2$ and $\nu$ (true anomaly) and in the planet's coordinate system by the angles $\theta$ and $\varphi$. 
Therefore, the orientation of the satellite in space is defined by three independent angles: $\Omega, \omega_{p}, I$. We introduce additionally two more parameters defining the shape and the size of the satellite's orbit: $a$ is the semimajor axis of the ellipse, $e$ is its eccentricity. Finally, the satellite's location on the orbit is determined by the parameter $t_{0}$. Therefore, we have six independent parameters defining satellite motion in space.

Since spherical function is an irreducible tensor, transforming it from the perigee coordinate system to the coordinate system associated with the planet yields

$$
Y_{l m}(\theta, \varphi)=\sum_{m^{\prime}} D_{m m^{\prime}}^{l *}\left(\Omega-\lambda-\frac{\pi}{2}, I, \omega_{p}+\frac{\pi}{2}\right) Y_{l m^{\prime}}\left(\frac{\pi}{2}, \nu\right) .
$$

Substituting this expression in (5.1) and using the fact that the unperturbed orbit has expression $R=\frac{a\left(1-e^{2}\right)}{1+e \cos \nu}=\frac{P}{1+e \cos \nu}$ ( $P$ is a focal parameter $)$, we will have an explicit expression of the force function of the non-spherical planet in terms of the Kepler's parameters

$$
v_{l}=\frac{f M}{P^{l+1}} I_{l_{0}}(1+\varepsilon \cos \nu)^{l+1} \sum_{m m^{\prime}} I_{l m} D_{l m}^{l *}\left(\Omega-\lambda-\frac{\pi}{2}, I, \omega_{p}+\frac{\pi}{2}\right) Y_{l m^{\prime}}\left(\frac{\pi}{2}, \nu\right) .
$$

For an axisymmetric planet one should take $m=0$; then

$$
\begin{aligned}
& v_{l}=\frac{f M}{P^{l+1}} I_{l_{0}}(1+\varepsilon \cos \nu)^{l+1} P_{l}(\cos \phi) \\
& \cos \phi=\sin I \sin \left(\omega_{p}+\nu\right)
\end{aligned}
$$

Therefore, we have expressed the force function in terms of Kepler elements.

In the unperturbed motion, the elements $a, e, \omega_{p}, \Omega, I$ are constants. They slowly evolve when the motion is perturbed. To find long-period terms, one has to average $V$ over $\tau$ because $\tau$ in unperturbed motion is not constant and is changing linearly with time.

However, the force function (5.3) depends on $\tau$ implicitly through the true anomaly $\nu$. Thus one can perform averaging over the period of revolution in the following way:

$$
\begin{aligned}
& \langle V\rangle=\frac{1}{2 \pi} \int_{0}^{2 \pi} V(\nu) d \tau=\frac{1}{2 \pi} \int_{0}^{2 \pi} V(\nu) \frac{d \tau}{d \nu} d \nu=\frac{1}{2 \pi} \int_{0}^{2 \pi} V(\nu) \frac{d \tau}{d t} \frac{d t}{d \nu} d \nu \frac{1}{2 \pi} \int_{0}^{2 \pi} V(\nu) n \frac{d t}{d \nu} d \nu \\
& =\frac{n}{\sqrt{\mu p}} \frac{1}{2 \pi} \int_{0}^{2 \pi} V(\nu) r^{2} d \nu=\frac{n p^{3 / 2}}{\sqrt{\mu}} \frac{1}{2 \pi} \int_{0}^{2 \pi} V(\nu)(1+e \cos \nu)^{-2} d \nu .
\end{aligned}
$$

In (5.5) the area integral $r^{2} \frac{d \nu}{d t}=\sqrt{\mu p}$ and the equation of unperturbed orbit were used.

Consider the eccentricity function

$$
S_{l m}(e)=\frac{1}{2 \pi} \int_{0}^{2 \pi}(1+e \cos \nu)^{l} e^{i m \nu} d \nu=\frac{l !}{(l+|m|) !} e^{|m|} x^{|m|-l} \frac{d^{|m|} P_{l}(x)}{d x^{|m|}} ; x=\left(1-e^{2}\right)^{-1 / 2} \text {. }
$$

Averaging the force function (5.3) using (5.5) yields

$$
\langle V\rangle=\frac{\sqrt{\mu} n}{P^{l-1 / 2}} \sum_{m, m^{\prime}} I_{l m} D_{m m^{\prime}}^{l}\left(\Omega-\lambda-\frac{\pi}{2}, I, \omega_{p}+\frac{\pi}{2}\right) Y_{l m^{\prime}}\left(\frac{\pi}{2}, 0\right) S_{l-1, m^{\prime}}(e) .
$$

Substituting (5.7) in the system of equations of motion in osculating variables [46, 47, one can see the orbit's semimajor axis does not evolve. If the planet's gravitational 
potential is axisymmetric, then (5.7) reduces to

$$
\langle V\rangle=\frac{\sqrt{\mu} n}{P^{l-1 / 2}} \sum_{m^{\prime}} I_{20} Y_{l-m^{\prime}}^{*}\left(I, \omega_{p}+\frac{\pi}{2}\right) Y_{l m^{\prime}}\left(\frac{\pi}{2}, 0\right) S_{l-1, m^{\prime}}(e) .
$$

In this case, the equations of motion have integrals $a=$ const, st $\sqrt{1-e^{2}} \cos I=$ const, $\left\langle V_{l}\right\rangle=$ const, and for some values of the index, $l$ can be integrated [47].

\section{Invariant expansion of the force function for spatial current distribu-}

tions. In this section we will obtain invariant expansion of the interaction energy for spatial distributions of electric currents, which demonstrates application of the vector addition theorem. Consider two spatial regions streamlined by quasi-stationary currents. The interaction energy of the two currents can be expressed by the integral

$$
V_{M}=\frac{\mu_{0}}{8 \pi} \int_{v_{1}} d v_{1} \int_{v_{2}} \frac{(\mathbf{j}(1) \cdot \mathbf{j}(2))}{\Delta} d v_{2}
$$

where $d v_{1}$ and $d v_{2}$ are the volume elements of spatial regions where currents $\mathbf{j}(1)$ and $\mathbf{j}(2)$ flow, and $\Delta$ is the distance between points of spatial current distributions. We denote the radius-vector of points in the volumes $v_{1}$ and $v_{2}$ with respect to their centers correspondingly as $\mathbf{r}_{1}$ and $\mathbf{r}_{2} ; \mathbf{R}$ will denote the radius-vector of the center of current distribution 2 with respect to the center of current distribution 1 . Then $\Delta=\mid \mathbf{r}_{1}-$ $\mathbf{a} \mid ; \mathbf{a}=\mathbf{r}_{2}+\mathbf{R}, r_{1}, r_{2}<R$, and $\mu_{0}$ is a magnetic constant.

Assuming $r_{1}<a$ we will use the expansion of the Green function for the vector Laplace equation [8],

$$
\frac{\mathbf{j}(1)}{\left|\mathbf{r}_{1}-\mathbf{a}\right|}=\sum_{l, L, q}\left(\mathbf{j}(1) \cdot \Im_{l q}^{L *}\left(\mathbf{r}_{1}\right)\right) \Re_{l q}^{L}(\mathbf{a}),
$$

where $\Im_{l q}^{L}(\mathbf{r})$ is a regular and $\Re_{l q}^{L}(\mathbf{r})$ is an irregular solid spherical vector corresponding to the expression (3.3) when $S=1$. Substituting (6.2) into (6.1) gives

$$
V_{M}=\frac{\mu_{0}}{8 \pi} \sum_{l, L, q} \mathfrak{M}_{l q}^{L}(1) \int_{v_{2}}(\mathbf{j}(2) \cdot \Re(\mathbf{a})) d v_{2},
$$

where

$$
\mathfrak{M}_{l q}^{L}(1)=\int_{v_{1}}\left(j(1) \cdot \Im_{l q}^{L}(\mathbf{r})\right) d v_{1}
$$

is a vector multipole moment.

In the expression for multipole moment (6.4), index $L$, due to the properties of a solid spherical vector $\Im_{l q}^{L}$, can take values $L=l, L=l+1, L=l-1$. We will show that a multipole moment is zero when $L=l-1$.

Taking into account that $\Im_{l q}^{l-1}(\mathbf{r})=\frac{1}{\sqrt{l(2 l-1)}} \nabla\left(\Im_{l q}(\mathbf{r})\right)$ when $L=l-1$ [8] and using the vector relation $\operatorname{div}(\mathbf{A} \cdot \varphi)=\varphi \operatorname{div} \mathbf{A}+\mathbf{A} \nabla \varphi$, we will have

$$
\begin{aligned}
\mathfrak{M}_{l q}^{l-1} & =\frac{1}{\sqrt{l(2 l+1)}} \int_{v}\left(\mathbf{j} \cdot \nabla\left(\Im_{l q}(\mathbf{r})\right)\right) d v \\
& =\frac{1}{\sqrt{l(2 l+1)}} \int_{v}\left[\operatorname{div}\left(\mathbf{j} \cdot \Im_{l q}(\mathbf{r})\right)-\Im_{l q}(\mathbf{r}) \operatorname{div} \mathbf{j}\right] d v .
\end{aligned}
$$


Changing in the first integral to the surface integration and noting that because of quasistatics $J_{n}=0$ and $\operatorname{div} j=0$, we will have $\mathfrak{M}_{l q}^{l-1}=0$.

We will now show that $\int_{v} \mathbf{j} \Re_{l q}^{l+1} d v=0$ when $L=l+1$. To do so, we notice that $\Re_{l q}^{l+1}=[(l+1)(2 l-3)]^{-\frac{1}{2}} \nabla\left(\Re_{l q}\right)$. Then, using similar reasoning, we will get the desired result. Therefore, by the virtue of proven, index $L$ in (6.3) takes only the single value $l$.

Consider now a vector multipole moment $\mathfrak{M}_{l q}^{l}$. Using the definition of solid spherical vector we will have, consequently,

$$
\left(\mathbf{j} \cdot \Im_{l q}^{l}\right)=\sum_{\mu, \sigma} C_{l \mu 1 \sigma}^{l q} \Im_{l \mu 1 \sigma} \cdot \mathbf{j}=\sum_{\mu, \sigma} C_{l \mu 1 \sigma}^{l q} \Im_{l \mu} j_{1 \sigma}=\left\{\Im_{l} \otimes j_{1}\right\}_{l q} .
$$

Substituting (6.5) into the integral (6.4) we will get an expression where the upper index may be omitted and thus the expression for a vector multipole moment will have the form

$$
\mathfrak{M}_{l q}=\int_{v}\left\{\Im_{l} \otimes j_{1}\right\}_{l q} d v .
$$

Assume that $l=1$ in $(\underline{6.6})$; then

$$
\mathfrak{M}_{1 q}=\int_{v_{1}}\left\{r_{1} \otimes j_{1}\right\}_{1 q} d v_{1}=i \sqrt{2} \mathfrak{M}_{1 q}
$$

which corresponds to the known expression for the vector of a full magnetic dipole moment of a system $\mathfrak{M}=\frac{1}{2} \int[\mathbf{r} \times \mathbf{j}] d v$.

To evaluate the integral in (6.3), we use the addition theorem (3.7) for a solid spherical vector $\Re_{l q}^{l}(\mathbf{a})$. After some manipulations we have

$$
\begin{aligned}
& \Re_{l q}^{l}(\mathbf{a})=\Re_{l q}^{l}\left(\mathbf{r}_{2}+\mathbf{R}\right)=-\sum_{n}\left[\sqrt{\frac{(2 l+2 n+1) ! l \cdot n}{(2 l+1) !(2 n) !(l+1)(n+1)}}\left\{\Im_{n}^{n}\left(\mathbf{r}_{2}\right) \otimes \Re_{l+n}(\mathbf{R})\right\}_{l q}\right. \\
& \left.+\sqrt{\frac{(2 l+2 n-1) !(l+n)}{(2 l+1) !(2 n-2) !(l+1) n}}\left\{\Im_{n}^{n-1}\left(\mathbf{r}_{2}\right) \otimes \Re_{l+n-1}(\mathbf{R})\right\}_{l q}\right] .
\end{aligned}
$$

Substituting (6.8) into (6.3) and noting that $\mathfrak{M}_{l q}^{l-1}=\mathfrak{M}_{l q}^{l+1}=0$, we will have an expression for the interaction energy of spatial current distributions

$$
V_{M}=-\frac{\mu_{0}}{8 \pi} \sum_{l, n} \sqrt{\frac{(2 l+2 n) ! l \cdot n}{(2 l+1) !(2 n) !(l+1)(n+1)}}\left(\mathfrak{M}_{l}(1) \cdot\left\{\mathfrak{M}_{n}(2) \otimes \Re_{l+n}(\mathbf{R})\right\}_{l}\right) .
$$

Using the change of coupling scheme for irreducible tensors, (6.9) can be transformed to the form

$$
V_{M}=-\frac{\mu_{0}}{8 \pi} \sum_{l, n}(-1)^{n+1} \sqrt{\frac{(2 l+2 n) ! l \cdot n}{(2 l+1) !(2 n) !(l+1)(n+1)}}\left(\left\{\mathfrak{M}_{l}(1) \otimes \mathfrak{M}_{n}(2)\right\}_{l+n} \cdot \Re_{l+n}(\mathbf{R})\right)
$$

Expression (6.9) may be considered as the interaction of vector multipoles of one current distribution with field, created by another current distribution. Expression (6.10) describes the interaction of vector multipoles of one current distribution with vector multipoles of another current distribution.

Expression (6.10), describing the interaction of two spatial current distributions, can be easily generalized on pair-wise interactions of $N$ spatial current distributions. Denote one of the interacting current distributions by index $k$ and the other by index $j$. 
Then, summing interactions, when $k$ and $j$ run over values from 1 to $N$ and noting that interactions $V_{k j}=V_{j k}$, we will have

$$
\begin{aligned}
& V_{M}=-\frac{\mu_{0}}{8 \pi} \sum_{\substack{k=0, k \neq j}}^{N-1} \sum_{j=0}^{N-1} \sum_{l, n}(-1)^{n+1} \sqrt{\frac{(2 l+2 n) ! l \cdot n}{(2 l) !(2 n) !(l+1)(n+1)}} \\
& \times\left(\left\{\mathfrak{M}_{l}(k) \otimes \mathfrak{M}_{n}(j)\right\}_{l+n} \cdot \Re_{l+n}\left(\mathbf{R}_{k j}\right)\right) .
\end{aligned}
$$

If we use (6.7), then the expression for energy (6.10) for the case $l=n=1$ will take the form

$$
\begin{aligned}
& V_{M}=-\frac{\mu_{0}}{4 \pi} \sqrt{6}\left(\left\{\mathfrak{M}_{1}(1) \otimes \mathfrak{M}_{1}(2)\right\}_{2} \cdot \Re_{2}(\mathbf{R})\right) \\
& =-\frac{\mu_{0}}{4 \pi} R^{-3}[(\mathfrak{M}(1) \cdot \mathfrak{M}(2))-3(\mathfrak{M}(1) \cdot \mathbf{R})(\mathfrak{M}(2) \cdot \mathbf{R})]
\end{aligned}
$$

which corresponds to dipole-dipole interaction. The field, generated by dipole $\mathfrak{M}(1)$, is expressed using irreducible tensors by the formula

$$
H_{1}=\frac{\mu_{0}}{4 \pi} \sqrt{10}\left\{\mathfrak{M}_{1}(1) \otimes \Re_{2}(\mathbf{R})\right\}_{1}
$$

and then (6.11) will take the known form of interaction of dipole $\mathfrak{M}_{1}(1)$ with dipole $\mathfrak{M}_{1}(2)$ :

$$
V_{M}=\left(H_{1} \cdot \mathfrak{M}_{1}\right)
$$

Similarly, one can consider the interaction of multipoles with higher order.

If a conductor carrying current is thin enough, then volume integration can be replaced with contour integration by substituting $\mathbf{j} d v \rightarrow I d \mathbf{l}$ in (6.4), where $I$ is the total current flowing though the conductor. Making this substitution in (6.6) we will have

$$
\mathfrak{M}_{l q}=I \int\left\{\Im_{l} \otimes d l_{1}\right\}_{l q} .
$$

We will apply this formula to determine the multipole moments of a loop of radius $b$ with current $I$. Assume that the z-axis of a Cartesian coordinate system associated with the loop coincides with the symmetry axis of the loop. Then axes $\mathrm{x}, \mathrm{y}$ will be in the plane of the loop. Determining cyclic projections of the vector $d l_{1 \mu}=i b \mu Y_{1 \mu}\left(\frac{\pi}{2}, \varphi\right) d \varphi$ and considering integral (6.14), we have

$$
\mathfrak{M}_{l q}=I b^{l+1} i \sum_{\mu, \nu} C_{l \nu 1 \mu}^{l q} Y_{l \nu}\left(\frac{\pi}{2}, 0\right) Y_{1 \mu}\left(\frac{\pi}{2}, 0\right) \int_{0}^{2 \pi} \mathrm{e}^{i(\mu+\nu) \varphi} d \varphi,
$$

which yields, after integration,

$$
\mathfrak{M}_{l 0}=2 \pi I i b^{l+1} Y_{l 1}\left(\frac{\pi}{2}, 0\right) .
$$

Knowledge of these multipole moments allows finding interaction energy of the loops when they are arbitrarily arranged.

7. Interaction of two spatial current loops. We will apply the results obtained in the previous section to find the force function for two interacting current loops. To find explicit dependence of the force function on angles, one has to express components of tensors $\mathfrak{M}_{l}$ in the source coordinate system through the components of the same tensor 


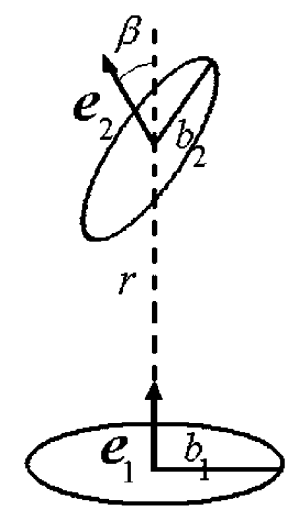

FIG. 2. Two current loops: the center of the arbitrarily oriented second loop is placed on the axis of the first loop

in the coordinate system related to the body, using the formula for irreducible tensors transformation

$$
\mathfrak{M}_{l q}=\sum_{q^{\prime}} \mathfrak{M}_{l q^{\prime}} D_{q q^{\prime}}^{l *}(\alpha, \beta, \gamma),
$$

where $\alpha, \beta, \gamma$ are the Euler's angles, characterizing the orientation of the body with respect to the chosen coordinate system.

We will associate the reference coordinate system with the first loop. Let the unit vector $\mathbf{e}_{1}$ be directed along the axis of the first loop, and let the unit vector $\mathbf{e}_{2}$ be directed along the axis of the second loop. Then, due to axial symmetry, the multipole moment for the first and second loops are correspondingly $\mathfrak{M}_{l q}(1)=\mathfrak{M}_{l 0}(1) Y_{l q}\left(\mathbf{e}_{1}\right)$ and $\mathfrak{M}_{l q}(2)=\mathfrak{M}_{l 0}(2) Y_{l q}\left(\mathbf{e}_{2}\right)$. Let the first loop have radius $a$ and the second loop have radius $b$. Then the expression for the interaction energy of the two loops (6.10) will be

$$
\begin{aligned}
& V_{M}=-\frac{\mu_{0}}{8 \pi} \sum_{l, n}(-1)^{n+1} \sqrt{\frac{(2 l+2 n) ! \cdot \cdot n}{(2 l) !(2 n) !(l+1)(n+1)}} \mathfrak{M}_{l 0}(1) \mathfrak{M}_{n 0}(2) \\
& \times\left(\left\{Y_{l}\left(\mathbf{e}_{1}\right) \otimes Y_{n}\left(\mathbf{e}_{2}\right)\right\}_{l+n} \cdot \Re_{l+n}(\mathbf{R})\right) .
\end{aligned}
$$

Consider a few particular cases:

(1) The loops are placed as shown on Fig. 2. The value for energy will be

$$
\begin{aligned}
& V_{M}=\frac{\mu_{0} \pi I_{a} I_{b}}{2} \sum_{l, n}(-1)^{n} \frac{(l+n) !}{l ! n !} \sqrt{\frac{l \cdot n}{(l+1)(n+1)}} \frac{a^{l+1} b^{n+1}}{r^{l+n+1}} \\
& \times Y_{l 1}\left(\frac{\pi}{2}, 0\right) Y_{n 1}\left(\frac{\pi}{2}, 0\right) P_{n}(\cos \beta) ; \quad \cos (\beta)=\left(\mathbf{e}_{1} \cdot \mathbf{e}_{2}\right) .
\end{aligned}
$$

(2) If the loops are placed in parallel as shown on Fig. 3, then expression (7.1) using the rule $\left\{Y_{l 1} \otimes Y_{n 1}\right\}_{l+n}=C_{l 1 n 1}^{l+n 1} Y_{l+n 1}$ [8] will take the form

$$
\begin{aligned}
& V_{M}=\frac{\mu_{0} \pi I_{a} I_{b}}{2} \sum_{l, n}(-1)^{n} \frac{(l+n) !}{l ! n !} \sqrt{\frac{l \cdot n}{(l+1)(n+1)}} \frac{a^{l+1} b^{n+1}}{r^{l+n+1}} \\
& \times Y_{l 1}\left(\frac{\pi}{2}, 0\right) Y_{n 1}\left(\frac{\pi}{2}, 0\right) P_{l+n}(\cos \gamma) ; \quad \cos (\gamma)=\left(\mathbf{e}_{1} \cdot \mathbf{e}_{r}\right) .
\end{aligned}
$$

(3) For the case shown on Fig. 4, the expression for the energy will be

$$
\begin{aligned}
& V_{M}=\frac{\mu_{0} \pi I_{a} I_{b}}{2} \sum_{l, n, q}(-1)^{n-q} \frac{(l+n) !}{l !} \sqrt{\frac{l \cdot n}{(l+1)(n+1)(n+q)(n-q)}} \frac{a^{l+1} b^{n+1}}{r^{l+n+1}} \\
& \times Y_{l 1}\left(\frac{\pi}{2}, 0\right) Y_{n 1}\left(\frac{\pi}{2}, 0\right) Y_{n q}\left(\mathbf{e}_{2}\right) Y_{l+n-q}\left(\mathbf{e}_{r}\right) .
\end{aligned}
$$




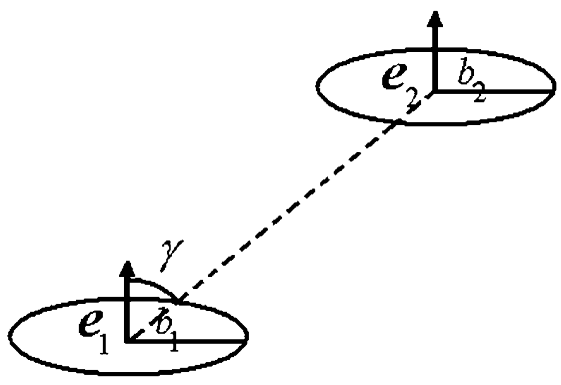

FIG. 3. Two current loops: the second loop is placed at an arbitrary point and is parallel to the first loop

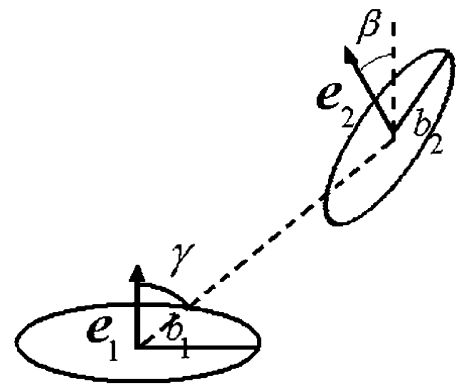

FIG. 4. Two current loops: the second loop is placed at an arbitrary point and has arbitrary orientation with respect to the first loop

8. Example: Calculation of multipole moments for permanent magnets with constant density of magnetic moment. We will give here one more representation for the integral $\mathfrak{M}_{l q}$, which is convenient for evaluating multipole moments for the interaction of permanent magnets with homogeneous density of magnetic moment $\lambda$. We have consequently

$$
\begin{aligned}
& \left(\mathbf{j} \cdot \Im_{l q}^{l}(\mathbf{r})\right)=-\frac{i}{\sqrt{l(l+1)}} r^{l-1}\left(\mathbf{j} \cdot[\mathbf{r} \times \nabla] Y_{l q}\right)=\frac{i}{\sqrt{l(l+1)}} r^{l-1}\left(\nabla Y_{l q} \cdot[\mathbf{r} \times \mathbf{j}]\right) \\
& =-\frac{i}{\sqrt{l(l+1)}}\left(\nabla\left(r^{l} Y_{l q}\right)[\mathbf{j} \times \mathbf{r}]\right)=-i \sqrt{\frac{2 l-1}{l+1}}\left(\Im_{l q}^{l-1} \cdot[\mathbf{j} \times \mathbf{r}]\right)=i \sqrt{\frac{2 l-1}{l+1}}\left\{\Im_{l-1} \otimes[\mathbf{j} \times \mathbf{r}]_{1}\right\}_{l q} .
\end{aligned}
$$

Substituting (8.1) in (6.4) and noting that $[\mathbf{j} \times \mathbf{r}] d v=2 \lambda d v$ gives

$$
\mathfrak{M}_{l q}=i \sqrt{\frac{2 l-1}{l+1}} \int\left\{\Im_{l-1} \otimes \lambda_{1}\right\}_{l q} d v .
$$

Expression (8.2) is convenient for calculation of the interaction for the bodies with homogeneous density of magnetization. In this case, $\lambda_{1}$ can be taken outside the integral, and we will have

$$
\mathfrak{M}_{l q}=i \sqrt{\frac{2 l-1}{l+1}}\left\{\lambda_{1} \otimes I_{l-1}\right\}_{l q}
$$

where $I_{l-1}=\int \Im_{l-1} d v$ is the irreducible inertia tensor of the rank $l-1$ with unit mass density. 
In the study [38], irreducible inertia tensors for bodies with various geometrical shape were calculated. They can be used to find interaction for magnets of various geometrical shapes.

9. The motion of a space vehicle in the terrestrial gravitational and magnetic fields. As a last example, consider the motion of a space vehicle in both gravitational and magnetic fields of the earth. If one accounts only for potential components of the external torque acting on a space vehicle in terrestrial gravitational and magnetic fields, then the force function creating these torques can be written as

$$
V=\frac{f m_{2}}{R^{3}}\left(I_{2} \cdot Y_{2}(\hat{\mathbf{R}})\right)+\left(\mathfrak{M}_{1} H_{1}\right)+\sqrt{\frac{3}{2}}\left(\mathfrak{L}_{2} \cdot\left\{H_{1} \otimes H_{1}\right\}_{2}\right) .
$$

Here the first term describes the force function of the gravitational torques, the second term describes magnetic torques caused by the terrestrial magnetic field and permanent magnetization of the space vehicle, and the third term describes the force function of magnetic moments caused by the magnetization of the vehicle's coating by an external magnetic field.

It is assumed that the terrestrial magnetic field $H_{1}$ is created by a magnetic dipole; its orientation in general does not coincide with the Earth's rotation axis. $H_{1}$ can be expressed analytically in terms of irreducible tensors as

$$
H_{1}=-\sqrt{10} \frac{\mu_{E}}{R^{3}}\left\{g_{1} \otimes Y_{2}(\hat{\mathbf{R}})\right\}_{1},
$$

where $g_{1}$ is a unit vector aligned along the Earth's magnetic dipole, $\hat{\mathbf{R}}$ is a unit vector along the radius-vector of the orbit $\mathbf{R}$, and $\mu_{E}$ is the magnitude of the Earth's dipole magnetic moment $\left(\mu_{E}=8 \cdot 10^{25} \mathrm{Oe}^{\cdot} \mathrm{cm}^{3}\right)$. Orientation of the dipole is defined by the coordinates $\varphi_{0}=11.7$ degrees, $\lambda_{0}=-69.6$ degrees, $H_{0}=\frac{\mu_{E}^{2}}{R_{E}} \cong 30824 \gamma\left(1 \gamma=10^{-5} \mathrm{Oe}\right)$, and $R_{E}$ is the Earth's radius.

Using the coupling scheme for irreducible tensors [8], $\left\{H_{1} \otimes H_{1}\right\}_{2}$ can be rewritten in the form

$$
\begin{aligned}
& \left\{H_{1} \otimes H_{1}\right\}_{2}=\sqrt{\frac{2}{3}} \frac{\mu_{E}^{2}}{R^{6}}\left[Y_{2}(\hat{\mathbf{R}})+\frac{1}{5} Y_{2}(\hat{\mathbf{m}})+\sqrt{\frac{2}{7}}\left\{Y_{2}(\hat{\mathbf{m}}) \otimes Y_{2}(\hat{\mathbf{R}})\right\}_{2}\right. \\
& \left.+\frac{54}{5} \sqrt{\frac{2}{7}}\left\{Y_{2}(\hat{\mathbf{m}}) \otimes Y_{4}(\hat{\mathbf{R}})\right\}_{2}\right] .
\end{aligned}
$$

Substituting (9.2) and (9.3) in (9.1) yields an invariant form of force function, where tensors $I_{2}, \mathfrak{M}_{1}, \mathfrak{L}_{2}$ characterize properties of the space vehicle's body, tensors $Y_{l}(\hat{\mathbf{R}})$ characterize the position of the center of mass of the space vehicle, and tensors $Y_{l}(\hat{\mathbf{m}})$ characterize the orientation of the Earth's magnetic dipole. This representation of force functions significantly simplifies the averaging procedure used to find the averaged equations.

The problem of motion of a space vehicle in terrestrial gravitational and magnetic fields was considered in [48] with the following assumptions: the space vehicle is dynamically symmetric, the magnetization is aligned along the vehicle's dynamic symmetry axis, and 
the vehicle's coating is magnetized along its symmetry axis. In the following we don't use these conditions.

We will express the force function in terms of phase variables of the rotary motion of the space vehicle in the perigee coordinate system. We will use the magnitude of the kinetic moment $\mathrm{K}$ and angles $\rho$ and $\sigma$, characterizing the orientation of the kinetic moment with respect to the perigee coordinate system, as phase variables. The orientation of the body with respect to the coordinate system of the kinetic moment will be defined by the Euler angles $\alpha, \beta, \gamma$.

Since the tensors $I_{2}, \mathfrak{M}_{1}, \mathfrak{L}_{2}$ are related to the body of the space vehicle, then transforming them to the perigee coordinate system yields

$$
\begin{aligned}
& V=f M R^{-3} \sum_{n m q} I_{2 n}^{*} Y_{2 n}(\hat{\mathbf{R}}) D_{m q}^{2}(\sigma, \rho, 0) D_{q n}^{2}(\alpha, \beta, \gamma) \\
& +\sum_{n m q} \mathfrak{M}_{1 n}^{*} H_{1 m} D_{m q}^{1}(\sigma, \rho, 0) D_{n q}^{1}(\alpha, \beta, \gamma) \\
& +\sqrt{3 / 2} \sum_{n m q} \mathfrak{L}_{2 n}^{*}\left\{H_{1} \otimes H_{1}\right\}_{2 m} D_{m q}^{2}(\sigma, \rho, 0) D_{n q}^{2}(\alpha, \beta, \gamma) .
\end{aligned}
$$

In the following it is assumed that there are no resonant relations between frequencies. Then the force function can be independently averaged over the free Euler-Poinsot motion, over the motion of the center of mass of the vehicle, and over the rotary motion of the earth [46]. The general form of the averaged force function will be

$$
\langle V\rangle=\frac{1}{2}(\mathbf{k}, B \mathbf{k})+(B \mathbf{k})
$$

where $B$ is a symmetric matrix; its coefficients are given in [46].

The averaged force function is the sum of two homogeneous polynomials of first and second order, depending on the projections of the unit vector of kinetic moment $k$ on the axes of the perigee coordinate system. Coefficients of these polynomials depend on the orbit elements $I, \omega_{\Pi}, e, \theta_{0}$, determining the orientation of the magnetic dipole relative to the earth's rotation axis, parameters characterizing inertial and magnetic properties of the space vehicle and initial conditions.

Using this averaged force function, one can study the dynamics of the space vehicle, in particular to find integrals determining the change of the vector of kinetic moment 46].

\section{REFERENCES}

[1] Bernard Friedman and Joy Russek, Addition theorems for spherical waves, Quart. Appl. Math. 12 (1954), 13-23. MR0060649 (15,702h)

[2] Seymour Stein, Addition theorems for spherical wave functions, Quart. Appl. Math. 19 (1961), 15-24. MR0120407 (22 \#11161)

[3] Orval R. Cruzan, Translational addition theorems for spherical vector wave functions, Quart. Appl. Math. 20 (1962/1963), 33-40. MR0132851 (24 \#A2687)

[4] M. Danos and L. C. Maximon, Multipole matrix elements of the translation operator, J. Mathematical Phys. 6 (1965), 766-778. MR0175515 (30 \#5699)

[5] M. N. Jones, "A Group-Theoretical Formulation of Geophysical Elastodynamics," Proc. R. Soc. Lond. A, vol. 356, pp. 549-568, 1977.

[6] F. Borghese, P. Denti, G. Toscano, and O. I. Sindoni, An addition theorem for vector Helmholtz harmonics, J. Math. Phys. 21 (1980), no. 12, 2754-2755, DOI 10.1063/1.524394. MR597591 (81m:33004) 
[7] B. U. Felderhof and R. B. Jones, Addition theorems for spherical wave solutions of the vector Helmholtz equation, J. Math. Phys. 28 (1987), no. 4, 836-839, DOI 10.1063/1.527572. MR880310 (88m:33018)

[8] D. A. Varshalovich, A. N. Moskalev, and V. K. Khersonskiĭ, Quantum theory of angular momentum, World Scientific Publishing Co. Inc., Teaneck, NJ, 1988. Irreducible tensors, spherical harmonics, vector coupling coefficients, $3 n j$ symbols; Translated from the Russian. MR 1022665 (90j:81062)

[9] E. Joachim Weniger and E. Otto Steinborn, A simple derivation of the addition theorems of the irregular solid harmonics, the Helmholtz harmonics, and the modified Helmholtz harmonics, J. Math. Phys. 26 (1985), no. 4, 664-670, DOI 10.1063/1.526604. MR785677 (86f:33010)

[10] E. J. Weniger, "Addition Theorems as 3-D Taylor Expansions," International Journal of Quantum Chemistry, vol. 76, pp. 280-295, 2000.

[11] Y. M. Urman, "Addition theorems for tensor spherical wave functions," Zh. Tekhn. Fiz., vol. 51, pp. 457-462, 1981.

[12] Ronald C. Wittmann, Spherical wave operators and the translation formulas, IEEE Trans. Antennas and Propagation 36 (1988), no. 8, 1078-1087, DOI 10.1109/8.7220. MR.962131(89j:78027)

[13] W. C. Chew, "A derivation of the vector addition theorem," Microwave and Optical Technology Letters, vol. 3, pp. 256-260, 1990.

[14] Daniel W. Mackowski, Analysis of radiative scattering for multiple sphere configurations, Proc. Roy. Soc. London Ser. A 433 (1991), no. 1889, 599-614, DOI 10.1098/rspa.1991.0066. MR1116968 (92h:78012)

[15] W. C. Chew and Y. M. Wang, "Efficient ways to compute the vector addition theorem," J. Electromagn. Waves Appl., vol. 7, pp. 661-665, 1993.

[16] K. T. Kim, "The translation formula for vector multipole fields and the recurrence relations of the translation coefficients of scalar and vector multipole fields," IEEE Trans. Antennas Propagat., vol. 44, pp. 1482-1487, 1996.

[17] Kristopher T. Kim, Efficient recursive generation of the scalar spherical multipole translation matrix, IEEE Trans. Antennas and Propagation 55 (2007), no. 12, 3484-3494, DOI 10.1109/TAP.2007.910358. MR2433218

[18] K. T. Kim, "Symmetry relations of the translation coefficients of the scalar and vector spherical multipole fields," Progress In Electromagnetics Research B, vol. 48, pp. 45-66, 2004.

[19] V. Rokhlin, "Diagonal Form of Translation Operators for the Helmholtz Equation in Three Dimensions, Research Report YALEU/DCS/RR-894, Dept. of Comp. Sci.," Yale University, New Haven, CT, 1992.

[20] Michael A. Epton and Benjamin Dembart, Multipole translation theory for the three-dimensional Laplace and Helmholtz equations, SIAM J. Sci. Comput. 16 (1995), no. 4, 865-897, DOI 10.1137/0916051. MR 1335895 (96f:35023)

[21] W. C. Chew, Vector addition theorem and its diagonalization, Commun. Comput. Phys. 3 (2008), no. 2, 330-341. MR2389804(2009c:78014)

[22] B. He and W. C. Chew, Diagonalizations of vector and tensor addition theorems, Commun. Comput. Phys. 4 (2008), no. 4, 797-819. MR2463190(2009j:43010)

[23] T. J. Dufva, et al., "Unified derivation of the translational addition theorems for the spherical scalar and vector wave functions," Progress In Electromagnetics Research B, vol. 4, pp. 79-99, 2008.

[24] W. Z. Yan, et al., "On the Convergency Properties of Translational Addition Theorems," Progress In Electromagnetics Research Symposium, Beijing, China, March 23-27, 2009.

[25] B. He and W. C. Chew, "The Tensor Addition Theorem: From the Viewpoint of Group Theory," presented at the Antennas and Propagation Society International Symposium, 2008. AP-S 2008. IEEE San Diego, CA, 2008.

[26] Victor Twersky, Multiple scattering by arbitrary configurations in three dimensions, J. Mathematical Phys. 3 (1962), 83-91. MR0142299 (25 \#5692)

[27] E. A. Ivanov, Two-body Diffraction of Electromagnetic Waves [in Russian]. Minsk: Nauika I Tekhnika, 1968.

[28] F. Borghese, P. Denti, R. Saija, G. Toscano, and O. I. Sindoni, Use of group theory for the description of electromagnetic scattering from molecular systems, J. Opt. Soc. Amer. A 1 (1984), no. 2, 183-191, DOI 10.1364/JOSAA.1.000183. MR736077 (85b:78021)

[29] F. Borghese, et al., Scattering from model nonspherical particles, Springer, 2007. 
[30] B. He and W. C. Chew, "Addition theorem", in Modeling and computations in electromagnetics, Lect. Notes Comput. Sci. Eng., vol. 59, Springer, Berlin, 2008, pp. 203-226, DOI 10.1007/978-3540-73778-0_8. MR2766831 (2012e:78017)

[31] P. A. Martin, Multiple scattering, Encyclopedia of Mathematics and its Applications, vol. 107, Cambridge University Press, Cambridge, 2006. Interaction of time-harmonic waves with $N$ obstacles. MR:2259988(2007k:35348)

[32] N. A. Gumerov and R. Duraiswami, Fast multipole methods for the helmholtz equation in three dimensions, Elsevier, 2004.

[33] N. A. Gumerov and R. Duraiswami, "Fast, Exact, and Stable Computation of Multipole Translation and Rotation Coefficients for the 3-D Helmholtz Equation," UMIACS TR, vol. 44, 2001.

[34] L. Greengard and V. Rokhlin, A fast algorithm for particle simulations, J. Comput. Phys. 73 (1987), no. 2, 325-348, DOI 10.1016/0021-9991(87)90140-9. MR918448 (88k:82007)

[35] Leslie Greengard and Vladimir Rokhlin, A new version of the fast multipole method for the Laplace equation in three dimensions, Acta numerica, 1997, Acta Numer., vol. 6, Cambridge Univ. Press, Cambridge, 1997, pp. 229-269, DOI 10.1017/S0962492900002725. MR1489257 (99c:65012)

[36] S. K. Veerapaneni, et al., "A fast algorithm for simulating vesicle flows in three dimensions," 2011.

[37] I. Lashuk, et al., "A massively parallel adaptive fast-multipole method on heterogeneous architectures," in Proceedings of the Conference on High Performance Computing Networking Storage and Analysis SC 092009.

[38] M. Harper Langston, Leslie Greengard, and Denis Zorin, A free-space adaptive FMM-based PDE solver in three dimensions, Commun. Appl. Math. Comput. Sci. 6 (2011), no. 1, 79-122, DOI 10.2140/camcos.2011.6.79. MR.2836694(2012k:65165)

[39] J. B. Minster, "Transformation of multipolar source fields under a change of reference frame," Geophys. J. R. Astr. Soc., vol. 47, pp. 397-409, 1976.

[40] Y. M. Urman, "Irreducible Tensors and Their Application in Problems of Dynamics of solids", Mechanics of solids, vol. 42, pp. 52-68, 2007.

[41] Willard Miller Jr., Symmetry and separation of variables, Addison-Wesley Publishing Co., Reading, Mass.-London-Amsterdam, 1977. With a foreword by Richard Askey; Encyclopedia of Mathematics and its Applications, Vol. 4. MR0460751 (57 \#744)

[42] Yu. M. Urman, Invariant expansion of the force function of mutual attraction for a system of bodies, Astronom. Zh. 66 (1989), no. 5, 1081-1092 (Russian); English transl., Soviet Astronom. 33 (1989), no. 5, 556-561 (1990). MR1053690(91c:70026)

[43] A. A. Orlov, An approximate representation of the potential of mutual attraction between two bodies, Vestnik Moskov. Univ. Ser. III Fiz. Astronom. 1960 (1960), no. 3, 69-76 (Russian). MR0124523 (23 \#A1835)

[44] "NIMA Technical Report TR8350.2," Department of Defense World Geodetic System, 1984.

[45] (2011). ESA news: Earth's gravity revealed in unprecedented detail Available: http://www.esa.int/esaCP/SEM1AK6UPLG_index_0.html

[46] Y. M. Urman, "Application of the method of irreducible tensors to celestial mechanics problems," Astronomy Reports, vol. 39, pp. 531-538, 1995.

[47] V. K. Abalakin, et al., Handbook on Celestial Mechanics and Astrodynamics. Moscow: Nauka, 1976.

[48] V. V. Beletskiy and A. A. Hentov, Rotary motions of a magnetized satellite. Moscow: Nauka, 1985. 